\title{
Erratum to: Predictors of Disapproval toward "Hard Drug" Use among High School Seniors in the US
}

\author{
Joseph J. Palamar
}

Published online: 6 February 2014

(C) Society for Prevention Research 2014

\section{Erratum to: Prev Sci DOI 10.1007/s11121-013-0436-0}

The original version of this article unfortunately contained a systematic error in the reporting of unadjusted odds ratios (ORs) for some variables reported in Tables 3-5. Although these updated ORs do not affect the overall outcomes of the study (which are determined by the adjusted ORs), the author has provided the corrected ORs in the table below along with an updated corresponding section in the Results:

With respect to disapproval towards use of powder cocaine (Table 3), identifying as a female or religious was associated with increased odds of disapproval, and cigarette, alcohol, marijuana and hard drug use decreased the odds of disapproval in unadjusted models. In the adjusted model, however, both alcohol use and marijuana use $(\mathrm{AOR}=1.05,99 \% \mathrm{CI}=0.80-1.38)$ lost significance. In the adjusted model, identifying as black decreased the odds of disapproval. Regarding crack disapproval (Table 3), identifying as female or religious, increased odds for disapproval towards crack use, and use of alcohol, cigarettes, or hard drugs, reduced odds of disapproval in unadjusted models. In the adjusted model, however, the significance of alcohol disappeared, and marijuana use remained non-significant $(\mathrm{AOR}=1.20$, $99 \% \mathrm{CI}=0.88-1.62)$. In the adjusted model, identifying

The online version of the original article can be found at http://dx.doi.org/ 10.1007/s11121-013-0436-0.

\section{J. J. Palamar $(\bowtie)$}

Department of Population Health, New York University Langone,

Medical Center, One Park Avenue, 7th Floor, New York, NY 10016,

USA

e-mail: joseph.palamar@nyu.edu as black decreased odds for disapproval, and use of only one hard drug was not associated.

With regard to LSD disapproval (Table 4), identifying as female or religious increased the odds for disapproval, and identifying as black, and alcohol, cigarette, marijuana and hard drug use decreased the odds of disapproval. In the adjusted model, identifying as black and alcohol use lost significance, and marijuana use $(\mathrm{AOR}=$ $0.58,99 \% \mathrm{CI}=0.40-0.84, p<.001)$ decreased odds for LSD disapproval. The odds of heroin disapproval (Table 4) were increased by identifying as a female or religious, and those who used alcohol, cigarettes or hard drugs were at lower odds for disapproval. However, in the adjusted model, the significance of sex (female) and alcohol use disappeared. Marijuana (AOR $=1.00,99 \%$ $\mathrm{CI}=0.59-1.70)$ and use of a single hard drug were not significant.

The odds of amphetamine disapproval (Table 5) were increased by identifying female, Hispanic or religious, and identifying as black, residing in a small MSA, and use of alcohol, cigarettes, marijuana or hard drugs lowered odds for disapproval. In the adjusted model, however, the significance of identifying as black, Hispanic or female was lost, as was the association for alcohol use; and high parent education, residing in a small MSA, and marijuana use lowered odds for disapproval $(\mathrm{AOR}=0.60,99 \% \mathrm{CI}=$ $0.41-0.88, p<.001)$. Finally, the odds for ecstasy disapproval (Table 5) were increased by identifying as female, Hispanic or religious, and odds were reduced in those who resided in a small MSA, or used alcohol, cigarettes, marijuana or hard drugs in their lifetime. In the adjusted model, significance of identifying and Hispanic and alcohol use was lost. Identifying as black, high parent education and marijuana use $(\mathrm{AOR}=0.62,99 \% \mathrm{CI}=$ $0.44-0.87, p<.001)$ significantly decreased the odds for disapproval. 


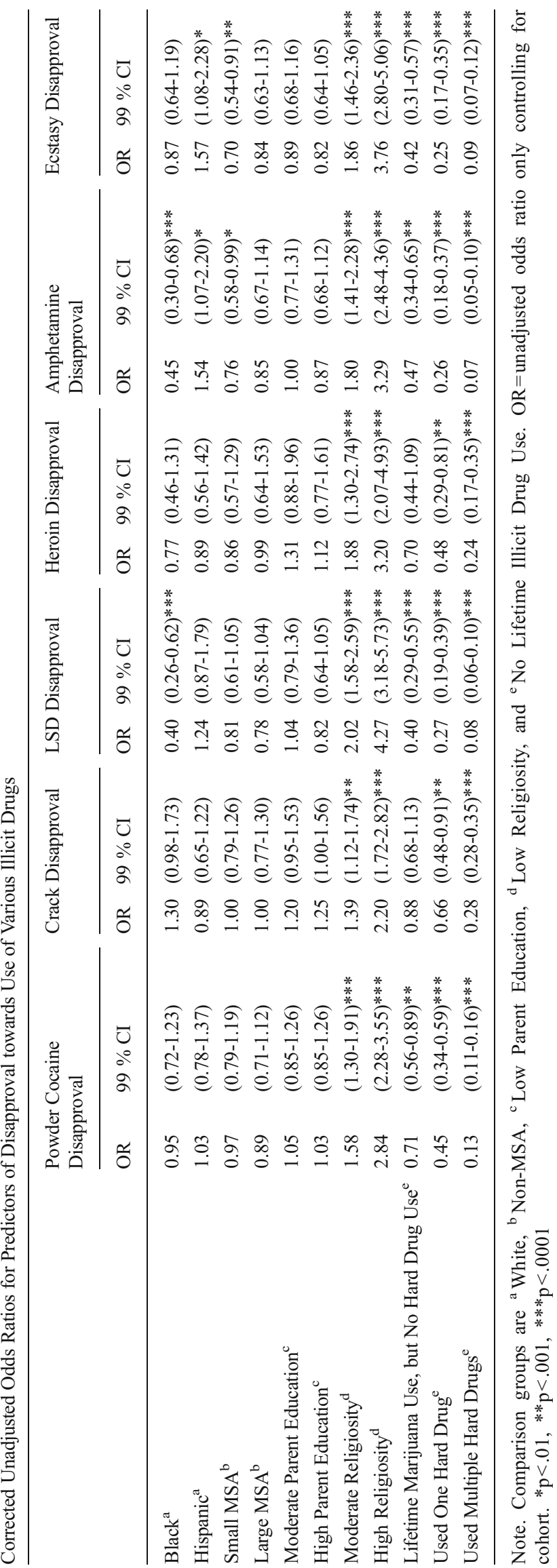

